\title{
Incidence in Ph-Negative Myeloproliferative Neoplasms in Armenia from 2005 to 2019
}

\author{
Sahakyan Lusine1 (1), Ter-Grigoryan Anahit1, Badikyan Maria², Saharyan Anahit ${ }^{2}$, \\ Shaljyan Alla ${ }^{2}$, Danelyan Samvel ${ }^{1,2}$
}

${ }^{1}$ Armenian Haematology Center aft.prof.R.Yeolyan, Yerevan, Armenain

${ }^{2}$ YSMU aft.M.Heratsi, Yerevan, Armenia

Email: lusisahakyan@gmail.com

How to cite this paper: Lusine, S., Anahit, T.-G., Maria, B., Anahit, S., Alla, S. and Samvel, D. (2020) Incidence in Ph-Negative Myeloproliferative Neoplasms in Armenia from 2005 to 2019. Open Journal of Epidemiology, 10, 355-368.

https://doi.org/10.4236/ojepi.2020.104029

Received: June 30, 2020

Accepted: September 6, 2020

Published: September 9, 2020

Copyright $\odot 2020$ by author(s) and Scientific Research Publishing Inc. This work is licensed under the Creative Commons Attribution International License (CC BY 4.0).

http://creativecommons.org/licenses/by/4.0/

\begin{abstract}
Introduction: Enhancements of laboratory diagnostics and the emergence of new therapies had a significant impact on the incidence, prevalence and survival of patients with myeloproliferative neoplasms (MPN). Published epidemiology data are scarce, and multiple sources are needed to assess the disease burden. The aim of our work was to identify the patterns and trends of incidence, prevalence and survival of patients with MPN in the Republic of Armenia (RA) for the period 2005-2019. Methods: The data from Hematology Center blood diseases register, Oncological Center cancer register, as well as the data from death registration were the basis of our research. Demographic data were obtained from National Statistical Office of RA. Results: Analysis of the data obtained has shown that during the reporting period the average annual incidence of MPN was 1.84 per 100.000 inhabitants, including 2.1 for males and 1.64 for females. Analysis of incidence rates of MPN in relation to sex and age in the period under study revealed high rates in patients in groups 65 - 74 (8.3) and 55 - 64 (5.12 per 100 thousand years), respectively. According to the data obtained in the group of patients with MPN, the high annual average incidence rates are noted in primary myelofibrosis (PMF) (1.09 in 2018) and polycythemia vera (PV) (0.89 in 2016), the lowest for essential thrombocythemia (ET) (0.7 in 2016) per 100.000 population, respectively. In comparing our data to those obtained for 1966-1971 and 1998-2004 periods, one may detect a statistically significant increase in the total incidence of PMF and PV ( $p<0.001)$. Conclusions: Analysis of the incidence rate in MPNs adjusted for age and gender shown the prevalence in group 65 74 (8.3) and in group 55 - 64 (5.13) per 100,000 inhabitants. The peak of incidence rate for both males and females was the age $65-74$ and the male female incidence ratio in this age group was 11.3:6.2. The increasing incidence rate in MPNs in Armenia depends on the improvement of laboratory
\end{abstract}


diagnosis. Thrombotic complications are observed in patients with an MPN in $45.3 \%$ of cases. In most cases, thrombosis is the first clinical symptom of an MPN, which determines the need for the introduction into clinical practice of molecular genetic testing methods among patients with thrombosis, an increase in blood levels, splenomegaly for the early diagnosis of clonal hematopoiesis and the use of a targeted drug.

\section{Keywords}

Ph-Negative Myeloproliferative Neoplasms, Incidence, Prevalence

\section{Introduction}

Improvement of laboratory diagnostics and the detection of new specific molecular abnormalities, such as Janus kinase 2 (Jak2 V617F), created the prerequisites for the revision of WHO 2008 diagnostic criteria, according to which several clonal Ph-negative neoplasms were combined into a group of chronic myeloproliferative diseases or neoplasms (MPN) [1] [2] [3] [4]. The most frequent MPN include polycythemia vera (PV), essential thrombocythemia (ET) and primary myelofibrosis (PMF). Less common conditions encompass systemic mastocytosis, chronic eosinophilic leukemia, chronic myelomonocytic leukemia, and chronic neutrophilic leukemia. The origin of MPN is associated with the transformation of the hematopoietic stem cell, resulting in excessive production of mature cells of erythroid, megakaryocytic and granulocyte lineages with a relatively long course of the disease [5] [6] [7]. In contrast to chronic myeloid leukemia, these diseases are characterized by the absence of a specific genetic marker: the Philadelphia chromosome $(\mathrm{Ph})-\mathrm{t}(9 ; 22)(\mathrm{q} 34 ; \mathrm{q} 11)$ and the fusion BCR-ABL gene [1] [5]. According to the literature data, there is a certain risk of family clustering of MPN [8] [9].

In the WHO system, patients with Ph-negative CML may be correctly classified as atypical CML (aCML) or, for cases that do not fit into any of these categories, MDS/MPD-unclassified. Collectively, these entities plus systemic mastocytosis (SM) are often referred to as "atypical" or "non-classic" MPD to distinguish them from the four "classical" MPD.

$\mathrm{PV}$, the most common MPD, originates from a single hematopoietic stem cell precursor. The principal molecular mechanism by which the abnormal PV clone develops and propagates is based on the ability of PV progenitor cells to form erythroid colonies in the absence of exogenous erythropoietin (Epo). In addition, the erythroid clone is hypersensitive to stimulating cytokines, such as insulin-like growth factor-1 or, to a lesser degree, interleukin-3 and granulocyte macrophage colony-stimulating factor, suggesting that this propagation occurs because of transcriptional dysregulation affecting a number of proteins involved in cytokine receptor signaling. Many pathophysiologic mechanisms of the molecular mechanisms of PV: the absence of an alternative product of the Epo re- 
ceptor (EpoR) gene, which inhibits EpoR-controlled erythropoiesis; overexpression of the PRV-1 gene, which belongs to the urokinase-type plasminogen activator receptor superfamily; deregulation of $\mathrm{Bcl}-\mathrm{x}$, which is an inhibitor of apoptosis; and increases of p16 protein and tyrosine phosphatase activity in red cell precursors [10].

Genome-wide scanning comparison of PV clonal and polyclonal cells from the same individuals revealed a loss of heterozygosity $(\mathrm{LOH})$ in chromosome $9 \mathrm{p}$, found in approximately $30 \%$ of PV patients. This $\mathrm{LOH}$ is not a result of a chromosomal deletion, but rather results from duplication of 1 parental allele and loss of the corresponding partner's allele, that is, uniparenteral disomy. Of note, the $9 \mathrm{p}$ region codes for the $\mathrm{p} 16$ and JAK2 tyrosine kinase gene [11]. The JAK family of protein tyrosine kinases is involved in cytokine receptor signaling, transmits the activating signal in the Epo-EpoR signaling pathway, and also is a required subunit for surface expression of cytokine receptors, including EpoR [12]. Ugo et al. have shown that inhibition of JAK2 abrogated Epo independence of erythroid progenitors [13]; this observation was followed by a description of the principal defect of the molecular basis of Ph-negative MPD, that is, a loss-of-function somatic mutation of an autoinhibitory JAK2 domain resulting from a valine-to-phenylalanine mutation at amino acid JAK2V617F. JAK2V617F leads to ongoing phosphorylation activity, which then can bind to a receptor and promote STAT recruitment and increased expression of PRV-1 [14]. This recruitment occurs in the absence of Epo [15]. This helps explain the known observed phenomenon of in vitro cytokine hypersensitivity. Furthermore, in a mouse bone marrow transplant model, murine marrow cells transduced with the JAK2 mutant allele led to a clinical phenotype consistent with PV, which included erythrocytosis, extramedullary hematopoiesis, and marrow fibrosis. This seminal work was rapidly confirmed by multiple groups, all of which demonstrated that $>90 \%$ of patients with PV and lower but still significant proportions of patients with ET and IMF carry the JAK2V617F mutation [16]. However, contradictory data exist as to whether or not this mutation is classically dominant. A study of 193 patients without 9p LOH found that 66 of 193 were heterozygous and 127 did not have the JAK2V617F mutation [17], findings similar to other reports, which also confirmed that approximately $30 \%$ of PV patients were homozygous for JAK2V617F [14].

The JAK2V617F mutation also appears to play a role in IMF and ET. Approximately $50 \%-70 \%$ of ET patients and $>50 \%$ of IMF patients have the JAK2V617F somatic mutation [18]. Presumably, this JAK2 mutation leads to a proliferation of clonal HSCs in these disorders through similar mechanisms as found in PV; however, the exact processes are not clear. Also, the fact that many of these patients do not have the JAK2V617F mutation raises other significant questions regarding etiology, specifically, what other mutations may exist that lead to these phenotypes in patients in whom the JAK2V617F mutation is not present. In this context, another important somatic mutation was identified in the transmembrane domain of the thrombopoietin receptor (cMPL) in 9\% 
(4/45) patients with JAK2V617F-negative IMF. The murine model of this mutation, but not the wild-type MPL, led to a fully penetrant MPD characterized by marked thrombocytosis, marked splenomegaly, and increased reticulin fibrosis. Interestingly, and significantly, a small-molecule JAK inhibitor, in vitro, inhibited MPLW515L-mediated proliferation and JAK-STAT signaling. This recent work demonstrated that this mutation, MPLW515L, also activates the JAK-STAT signaling pathway, and that the likely presence of multiple other somatic and/or germ-line mutations besides JAK2V617F and MPLW515L will activate this signaling cascade and may account for the cases of JAK2V617F-negative IMF and ET. However, whether the JAK2V617F mutation is the PV disease initiating mutation is not clear; some PV JAK2V617F-negative patients have monoclonal hematopoiesis and Epo-independent erythropoiesis, and in some individual PV families there are PV subjects with and without the JAK2V617F mutation.

From a clinical point of view, patients with MPD have a more aggressive clinical course, poorer response to treatment, and shorter survival than those with typical CML. In a series of 76 cases from a single institution over a 32-year period, the median overall survival was only 2 years, with a median age at presentation of 66 years. Of those who died, one-third had transformed to myeloid blast crisis; the remainder died from other causes. Multivariate analysis indicated that age greater than 65 years, hemoglobin $<10 \mathrm{~g} / \mathrm{dl}$, and leukocyte count $>50 \times$ $109 / 1$ were independent predictors of a poor prognosis and that these factors could be used to distinguish a high-risk group with a median survival of only 9 months from a lower-risk group with a median survival of 38 months [19].

Enhancements of laboratory diagnostics and the emergence of new therapies had a significant impact on incidence, prevalence and survival of patients with MPN. Published epidemiology data are scarce, and multiple sources are needed to assess the disease burden. The purpose of our study was to identify the patterns and trends of incidence, prevalence and survival of patients with MPN in the Republic of Armenia for the period 2005-2019.

\section{Material and Methods}

\section{Patients and Samples}

The data from Hematology Center blood diseases register, Oncological Center cancer register, as well as the data from death registration were basis of our research. The current study includes 826 patients with MPD diagnosed according to WHO 2008 diagnostic Criteria [20] at Armenian Hematology Center aft. prof. R. Yeolyan between 2005 and 2019. The patients' diagnose were established mainly by histological analysis of bone marrow. In some cases, genetic analysis for JAK2V617F mutation was performed. The limitation of the genetic analysis was due to the fact that JAK2V617F mutation testing was not available in our center for most of the time of our study period. Treatment was done with hydroxyurea, some patients with PV were treated with alpha-interferon or Ruxolitinib. 
Demographic data were obtained from the National Statistical Office PA (http://www.armstat.am). The calculation of standardized indicators has been based upon the data from the Demographic compendium of Armenia from 2005 to 2019. All $\mathrm{p}$ values were two tailored and the level of significance was set at the level of $\mathrm{p}<0.05$. Continuous variables were shown as median and range. Categorical variables were described as frequency and percentage. Comparison between categorical variables was performed by Chisquared statistics. Comparison between categorical and continuous variables was performed by Mann-Whitney U-test or Kruskal-Wallis test, where appropriate. All parameters used for statistical analysis, except for those addressing prognosis (survival, leukemic or fibrotic transformation), were those obtained at the time of diagnosis and before any therapeutic intervention.

\section{Result and Discussion}

During the study period (2005-2019), 826 primary patients with MPN were registered and/or treated in the Hematological Center of Armenia, of which 436 (52.8\%) male and $390(47.2 \%)$ female. Of the total number of patients with MPN, 91 people (63.7\% female) were with ET, 335 with PV (45.4\% female), 360 with PMF (45.6\% female) and 31 with other Ph-negative MPNs (35.4\% female).

These patients' clinical and biological characteristics are summarized in Tables 1-3. For each described parameter, we also give the number and the percentage of data available in the published population. The median age at diagnosis was $47.7 \pm 2.7$ years for ET patients, $58.9 \pm 2.5$ years for PMF patients, and $55.4 \pm 3.1$ years for PV patients. The percentage of female cases was also different between the three groups (63.7\% in ET, 45.6\% in PMF and 54.6\% in PV).

The reason for the original consultation was unclear or unknown in most cases. At the time of the diagnosis, $65.9 \%$ of the ET patients, $54.4 \%$ of the PMF and $69.9 \%$ of PV were declared to be asymptomatic. Splenomegaly and hepatomegaly was the most frequent abnormal sign described in the papers: $50.5 \%$ and $45.1 \%$ of ET, $30.8 \%$ and $64.4 \%$ of PMF, $71.9 \%$ and $66.0 \%$ of PV patients had a palpable spleen.

Table 1. Demographic and clinical characteristics of patients with ET in Armenia.

\begin{tabular}{cccc}
\hline Feature & Median (range) & $\mathrm{N}$ & Rate, $\%$ \\
\hline Age, $\mathrm{y}$ & $47.7(18-76)$ & & \\
Sex, male/female & & $33 / 58$ & $36.3 / 63.7$ \\
Constitutional symptoms & & 60 & 65.9 \\
Palpable splenomegaly & & 46 & 50.5 \\
Palpable hepatomegaly & & 41 & 45.1 \\
Hemoglobin, $\mathrm{g} / \mathrm{l}$ & $141.6(75-188)$ & & \\
WBC, count, $\times 10^{9} / \mathrm{L}$ & $10.6(5-22.4)$ & & \\
Platelet count, $\times 10^{9} / \mathrm{L}$ & $1008.1(146-1843)$ & &
\end{tabular}


Table 2. Demographic and clinical characteristics of patients with PV in Armenia.

\begin{tabular}{cccc}
\hline Feature & Median (range) & $\mathrm{N}$ & Rate, $\%$ \\
\hline Age, $y$ & $55.4(21-78)$ & & \\
Sex, male/female & & $152 / 183$ & $45.4 / 54.6$ \\
Constitutional symptoms & & 234 & 69.9 \\
Palpable splenomegaly & & 241 & 71.9 \\
Palpable hepatomegaly & & 221 & 66.0 \\
Hemoglobin, g/l & $196.8(24.3-263)$ & & \\
WBC, count, $\times 10^{9} / \mathrm{L}$ & $13.9(3.69-69.3)$ & & \\
Platelet count, $\times 10^{9} / \mathrm{L}$ & $510.3(40-5178)$ & & \\
\hline
\end{tabular}

Table 3. Demographic and clinical characteristics of patients with PMF in Armenia.

\begin{tabular}{cccc}
\hline Feature & Median (range) & N & Rate, $\%$ \\
\hline Age, $y$ & $58.9(20-83)$ & & \\
Sex (male) & & $196 / 164$ & $54.4 / 45.6$ \\
Constitutional symptoms & & 196 & 54.4 \\
Palpable splenomegaly & & 111 & 30.8 \\
Palpable hepatomegaly & & 232 & 64.4 \\
Hemoglobin, g/l & $107.5(24-217)$ & & \\
WBC, count, $\times 10^{9} / \mathrm{L}$ & $13.9(0.74-61.15)$ & & \\
Platelet count, $\times 10^{9} / \mathrm{L}$ & $444.9(27-6975)$ & & \\
\hline
\end{tabular}

The results of the full blood count at diagnosis are presented in Tables 1-3. For ET patients, the median leukocyte count was $10.6 \times 10^{9} / \mathrm{L}$, the hemoglobin concentration was $141.6 \mathrm{~g} / \mathrm{L}$ and the platelet count was $1008.1 \times 10^{9} / \mathrm{L}$. For PMF patients, the median leukocyte count was $13.9 \times 10^{9} / \mathrm{L}$, the hemoglobin concentration was $107.5 \mathrm{~g} / \mathrm{L}$ and the platelet count was $444.9 \times 10^{9} / \mathrm{L}$. For PV patients, the median leukocyte count was $13.2 \times 10^{9} / \mathrm{L}$, the hemoglobin concentration was $180 \mathrm{~g} / \mathrm{L}$ (maximum level, $189 \mathrm{~g} / \mathrm{L}$ ), the maximum hematocrit was $72.5 \%$ and the platelet count was $799 \times 10^{9} / \mathrm{L}$.

According to the International Prognostic Scoring System (IPSS) (Table 4), $37.3 \%$ ET patients were in the low-risk group, $13.2 \%$ in the intermediate-1, $49.5 \%$ in the intermediate- 2 , and no one in the high risk category. In PV patients $97.9 \%$ were in the low-risk group, $2.1 \%$ in the intermediate- 1 group. $6.6 \%$ of PMF patients were in the low-risk group, and $93.4 \%$ in the intermediate-2 groups. In total $49.5 \%$ of MPN patients were in the low-risk group, $44.9 \%$ in the intermediate-1, $5.6 \%$ in the intermediate-2, and no one in the high risk category.

The Dynamic International Prognostic Scoring System (DIPSS) score was calculated at the time of last follow up. When DIPSS risk scores were applied, in ET group $19.5 \%$ patients were in the low-risk group, $21.5 \%$ in the intermediate-1, $60.1 \%$ in the intermediate-2, and no one in the high risk category. In PV patients $4.03 \%$ in the intermediate- $1,83.9 \%$ in the intermediate- 2 , and $11.7 \%$ in the high risk group. $1.0 \%$ of PMF patients were in the low-risk group, $37.1 \%$ in 
the intermediate-1, $57.5 \%$ in the intermediate- 2 , and $4.5 \%$ in the high risk group. In total $2.5 \%$ of MPN patients were in the low-risk group, $21.4 \%$ in the intermediate- $1,68.8 \%$ in the intermediate- 2 , and $7.3 \%$ in the high risk group.

The average annual incidence of MPN was 1.84 per 100.000 inhabitants, including 2.10 for male and 1.64 for female. Linear analysis of the average annual incidence indicators of the MPN (Figure 1) revealed a tendency to a certain increase in the incidence in the period under study, both for male and female.

In different years, there were higher rates for male $(2017,2018,2019)$, and female $(2018,2019)$. The overall incidence of MPN between male and female was 1.9:1.5, respectively. We also analyzed the incidence rates of patients with MPN (Table 5) in the three-year dynamics for the period 2005-2019. Comparison of incidence rates of MPN revealed a significant increase in them in 2017-2019 compared with 2005-2007 (2.59 against 1.12 per 100,000 population). In general, there were no specific patterns of incidence depending on sex and age.

According to the data received, the increase in the incidence of MPN is particularly noticeable in 2017-2019 and 2014-2016. The incidence of ET and PV in the period 2017-2019 prevails mainly in female (0.24 in male to 0.40 in female for ET and 0.83 in male to 0.96 in female for ET, respectively), whereas in other entities of the MPN, the prevalence of indicators in male is noticeably higher. This circumstance is confirmed by the data of other authors [3] [21] [22] [23].

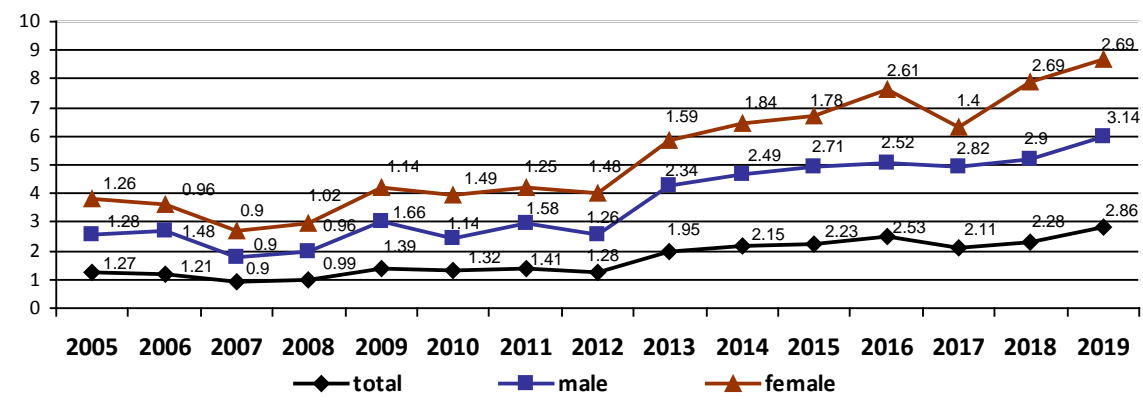

Figure 1. MPN incidence rates in Armenia during 2005-2019.

Table 4. Comparison of prognostic risk distribution.

\begin{tabular}{cccccc}
\hline \multirow{5}{*}{ IPSS risk } & & $\mathrm{ET} / \% /$ & $\mathrm{PV} / \% /$ & $\mathrm{PMF} / \% /$ & $\mathrm{MPN} / \% /$ \\
\hline & Low & $34 / 37.3$ & $328 / 97.9$ & $22 / 6.6$ & $409 / 49.5$ \\
& Intermediate - 1 & $12 / 13.2$ & $7 / 2.1$ & $312 / 93.4$ & $371 / 44.9$ \\
& Intermediate - 2 & $45 / 49.5$ & - & - & $46 / 5.6$ \\
& High & - & - & - & - \\
& Low & $16 / 19.5 /$ & - & $3 / 1.0$ & $21 / 2.5$ \\
\multirow{2}{*}{ DIPSS risk } & Intermediate - 1 & $16 / 21.5 /$ & $13 / 4.03$ & $124 / 37.1$ & $177 / 21.4$ \\
& Intermediate - 2 & $50 / 60.1 /$ & $251 / 83.9$ & $192 / 57.5$ & $568 / 68.8$ \\
& High & - & $35 / 11.7$ & $15 / 4.5$ & $60 / 7.3$ \\
\hline
\end{tabular}


Table 5. Incidence rates patients with MPN in dynamic during 2005-2019 (per 100,000 inhabitants).

\begin{tabular}{|c|c|c|c|c|c|c|}
\hline \multicolumn{2}{|c|}{ Nosology, sex } & \multirow{2}{*}{$\begin{array}{c}2005-2007 \\
1.12(0.9-1.27)\end{array}$} & \multirow{2}{*}{$\begin{array}{c}2008-2010 \\
1.23(0.99-1.38)\end{array}$} & \multirow{2}{*}{$\begin{array}{c}2011-2013 \\
0.49(0.22-1.85)\end{array}$} & \multirow{2}{*}{$\begin{array}{c}2014-2016 \\
1.98(1.67-2.15)\end{array}$} & \multirow{2}{*}{$\begin{array}{c}2017-2019 \\
2.59(2.11-2.86)\end{array}$} \\
\hline & total & & & & & \\
\hline \multirow{2}{*}{ MPN } & female & $1.14(0.90-1.56)$ & $1.28(1.02-1.43)$ & $1.26(0.13-1.40)$ & $1.19(0.83-1.53)$ & $2.24(1.40-2.69)$ \\
\hline & total & $0.02(0-0.03)$ & $0.03(0.03-0.03)$ & $0.12(0.09-0.15)$ & $0.50(0.20-0.98)$ & $0.32(0.13-0.50)$ \\
\hline \multirow[t]{3}{*}{ ET } & male & $0.02(0-0.06)$ & $0.02(0-0.06)$ & $0.13(0.06-0.19)$ & $0.16(0.07-0.21)$ & $0.24(0.14-0.36)$ \\
\hline & female & $0.02(0-0.06)$ & $0.04(0-0.06)$ & $0.12(0.12-0.13)$ & $0.38(0.19-0.57)$ & $0.40(0.10-0.64)$ \\
\hline & total & $0.49(0.44-0.56)$ & $0.66(0.62-0.71)$ & $0.56(0.44-0.96)$ & $0.80(0.70-0.87)$ & $1.1(0.84-1.25)$ \\
\hline \multirow[t]{2}{*}{ PV } & male & $0.51(0.26-0.77)$ & $0.66(0.45-0.82)$ & $0.63(0.44-0.96)$ & $0.95(0.83-1.18)$ & $1.3(1.0-1.5)$ \\
\hline & total & $0.62(0.43-0.81)$ & $0.53(0.34-0.65)$ & $0.79(0.64-0.89)$ & $0.98(0.83-1.10)$ & $0.96(0.91-1.0)$ \\
\hline \multirow[t]{3}{*}{ PMF } & male & $0.58(0.39-0.71)$ & $0.51(0.45-0.57)$ & $0.98(0.75-1.24)$ & $1.30(1.18-1.39)$ & $1.04(0.85-1.27)$ \\
\hline & female & $0.66(0.48-0.90)$ & $0.50(0.24-0.66)$ & $0.60(0.44-0.83)$ & $0.68(0.51-0.76)$ & $0.90(0.77-1.09)$ \\
\hline & total & - & $0.02(0-0.06)$ & $0.02(0-0.03)$ & $0.11(0.07-0.17)$ & $0.19(0.07-0.37)$ \\
\hline \multirow[t]{2}{*}{$\begin{array}{c}\text { Another } \\
\text { MPN }\end{array}$} & male & - & - & - & $0.19(0.07-0.28)$ & $0.26(0.07-0.64)$ \\
\hline & female & - & $0.04(0-0.11)$ & $0.04(0-0.06)$ & $0.04(0.0-0.06)$ & $0.11(0.07-0.13)$ \\
\hline
\end{tabular}

We tend to assume that in Armenia, as well as all over the world, with the improvement of the instrumentation of the diagnostic service, in particular with the extensive use of automated platelet count, the special attention of physicians was drawn to patients with their elevated rates, which is associated with such a sharp increase in the incidence rate MPN in recent years.

Analysis of incidence rates of MPN in relation to sex and age in the period under study revealed high rates in patients in groups $65-74$ (8.3) and $55-64$ (5.12 per 100 thousand years), respectively (Figure 2). In both sex male and female with MPN, the incidence peak is at the age of 65 - 74 years, the ratio of the incidence of male and female in this age group is 11.3: 6.2, respectively (Figure 2).

According to the data obtained in the group of patients with MPN, the high annual average incidence rates are noted in PMF (1.09 in 2018) and PV (0.89 in 2016), the lowest for ET (0.7 in 2016) per 100.000 population, respectively (Figure 3).

Comparison of incidence rates of patients with PMF and PV with similar, obtained on the basis of conducted studies (Table 6) in Armenia for 1966-1971 and 1998-2004, showed that there is a statistically significant increase in the total incidence of PMF (2.3 and 1.8 times) and PV (1.7 and 1.6 times) $(\mathrm{p}<0.001)$.

As for the indicators of ET, in previous studies in Armenia they were not covered. However, an analysis of the available literature [24] [25] revealed an increase in the incidence of ET after 2008 and other countries. Thus, in European 
Table 6. Comparison of incidence rates of PV and PMF during 2005-2019 with our early studies (1966-1971 and 1998-2004).

\begin{tabular}{cccc}
\hline Diseases & 1966-1974 & 1998-2004 & $2005-2019$ \\
\hline PV & 0.31 & 0.40 & 0.72 \\
& & & $\begin{array}{l}\left(\mathrm{p}_{1}<0.001\right) \\
\left(\mathrm{p}_{2}<0.001\right)\end{array}$ \\
& & & 0.78 \\
PMF & 0.45 & 0.47 & $\left(\mathrm{p}_{1}<0.001\right)$ \\
& & & $\left(\mathrm{p}_{2}<0.001\right)$ \\
\hline
\end{tabular}

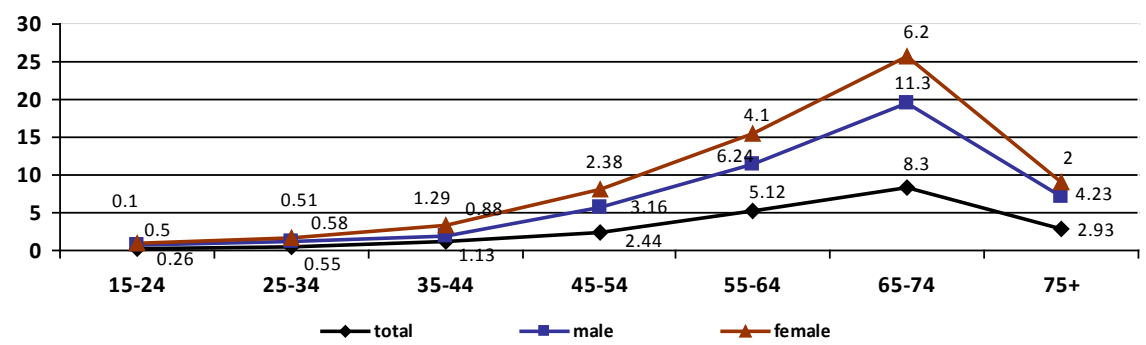

Figure 2. Incidence rates of patients with MPN depending on age and sex during 2005-2019 (per 100,000 inhabitants).

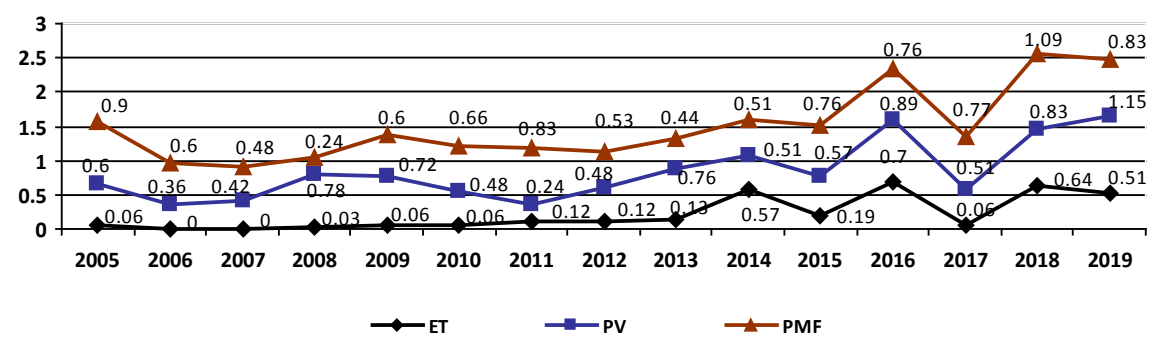

Figure 3. Incidence rates of patients with MPN depending on the type of diseases during 2005-2019 (per 100,000 inhabitants).

countries [26], according to the EMBASE/biomedical database/analysis of the three European registries, the incidence rates of the MPN have been varied: PMF $0.22-0.99$, PV $0.01-2.61$ and ET $0.21-2.27$ per 100,000 populations, respectively. The combined annual incidence rates for PV, ET, and PMF were 0.84, 1.03 , and 0.47 per 100,000. In Russia, the frequency of occurrence of PV $0.5-1.5$ cases, ET $0.6-2.1$ and PMF $0.72-1.56$ cases per 100,000 population is described [27].

Taking into account the clonal markers, mainly the JAK2-V617F mutation, as well as the lower platelet count $(450 \times 109 / \mathrm{L})$ in diagnosis, the frequency of ET after 2008 in different countries increased more than 2 times, both in male and in female [28] [29]. Reduction of the threshold value of platelets in the recommendations of WHO 2008, had little impact on the incidence rates of the PMF and PV. The JAK2 V617F mutation is found in $90 \%$ - 95\% of cases of PV, $50 \%$ $70 \%$ of cases of ET and $40 \%-50 \%$ of cases of PMF [28]. A number of authors attach great importance of screening for the JAK2 mutation in PV, which has 
completely transformed the diagnostic approach to this disease: in current recommendations, when suspected of PV, screening of this mutation in peripheral blood in a set of diagnostic procedures is recommended [30]. Analysis of morbidity rates of ET, PV and PMF in Armenia, depending on sex and age, showed high rates in patients in groups of $65-74$ (0.46 for ET, 3.1 for PV, 4.4 for PMF per 100 thousand inhabitants) and 55 - 64 (0.45 for ET, 2.4 for PV per 100 thousand inhabitants) and 75+ (4.4 for PMF per 100 thousand inhabitants), respectively (Figure 2). In both female and male with MPN, the incidence peak is at the age of 65 - 74 years for all nosology (Figures 4-6).

Thus, for the study period, the average annual incidence of the MPN was 1.35 per 100,000 inhabitants, including 1.45 for male and 1.25 for female. The incidence rates of MPN increased markedly in 2014-2016. The incidence of ET in the period 2014-2016 prevailed mainly in females, whereas, in other entities of MPN, there were high rates in males.

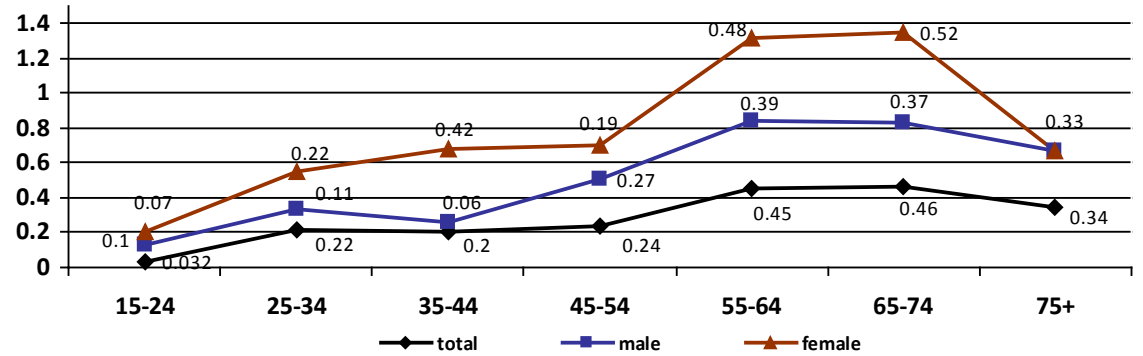

Figure 4. Incidence rates of patients with ET depending of age and sex during 2005-2019 (per 100,000 inhabitants).

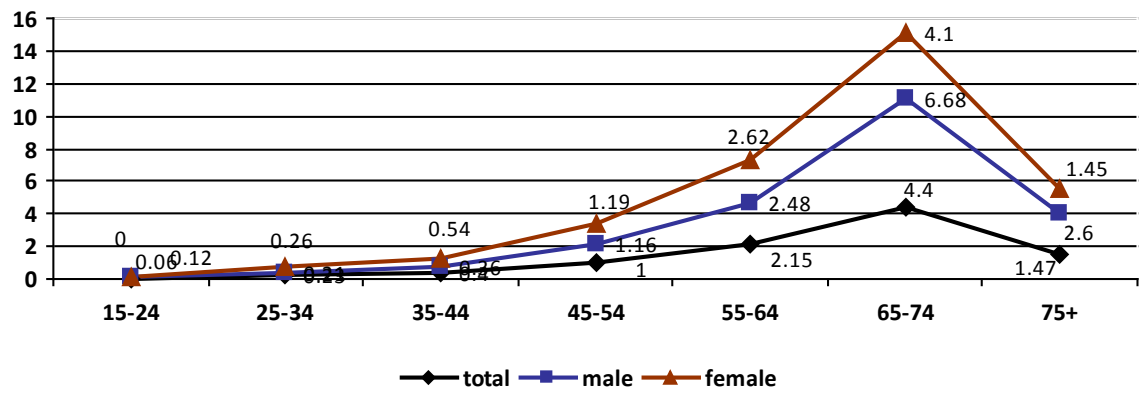

Figure 5. Incidence rates of patients with PMF depending of age and sex during 2005-2019 (per 100,000 inhabitants).

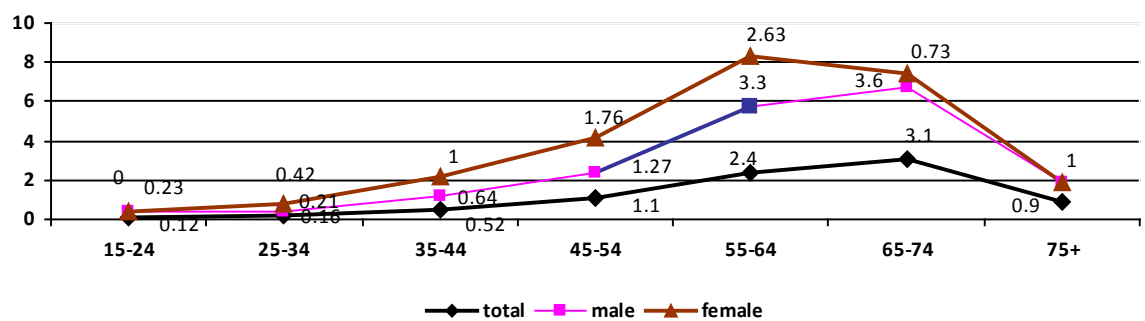

Figure 6. Incidence rates of patients with PV depending of age and sex during 2005-2019 (per 100,000 inhabitants). 
Splenomegaly was detected in $30.5 \%$ (12/18) of patients with ET, in 48.5 (79/65) with PV, and in 60.2\% (109/92) with PMF. Thrombotic complications were recorded in $37.1 \%$ of patients with PV (11/18), 32\% (10/29) with ET and 26\% (27/60) with PMF. In 45.3\% (156/171) cases, thrombosis was the first clinical symptom of the disease. On average, the time from the moment of thrombosis to the diagnosis of the disease was 1 year.

Among thrombotic complications in patients with PV, arterial vascular thrombosis predominated-acute cerebrovascular accidents (25.8\%), myocardial infarction (4.8), lower/deep vein thrombosis (DVT) of the lower extremities (3.0) and less common visceral vein thrombosis (3.5\%). Thrombosis in patients with ET in $12.8 \%$ of cases is presented by stroke and myocardial infarction, in 6\% DVT. In patients with PMF, in $12.1 \%$ of cases there was a stroke, in 10.5 DVT and thrombosis of system v. portae/portal vein thrombosis, and in 3.9\% myocardial infarction. The median time interval between the diagnosis of the disease and the development of thrombosis was 3 years (from 0 to 30 years). Repeated episodes of thrombotic complications were observed in $17.2 \%$ of patients undergoing thrombosis.

Interferon preparations as the first line of therapy were received by $42.5 \%$ of patients (17/40) with PV, 51.3\% (20/39) with ET and 24\% (6/25) with PMF. Hydroxyurea was prescribed in 30\% (12/40) patients with PV, 23.1\% (9/39) with ET and $44 \%(11 / 25)$ with PMF. In other cases, antiplatelet therapy and vascular therapy was performed. 8 patients are currently receiving targeted therapy with positive dynamics of the drug Ruxolitinib. Thrombotic complications were not observed in patients treated with Ruxolitinib therapy.

\section{Conclusion}

Analysis of the incidence rate in MPNs adjusted for age and gender shown prevalence in group $65-74$ (8.3) and in group 55 - 64 (5.13) per 100,000 inhabitants. The peak of incidence rate for both males and females was the age 65 - 74 and the male:female incidence ratio in this age group was 11.3:6.2. The increasing incidence rate in MPNs in Armenia depends on the improvement of laboratory diagnosis. Thrombotic complications are observed in patients with MPN in $45.3 \%$ of cases. In most cases, thrombosis is the first clinical symptom of a myeloproliferative disease, which determines the need for the introduction into clinical practice of molecular genetic testing methods among patients with thrombosis, an increase in blood levels, splenomegaly for the early diagnosis of clonal hematopoiesis and the use of a targeted drug.

\section{Conflicts of Interest}

The authors declare no conflicts of interest regarding the publication of this paper.

\section{References}

[1] Brière, J.B. (2007) Essential Thrombocythemia. Orphanet Journal of Rare Diseases, 
2, Article No. 3. https://doi.org/10.1186/1750-1172-2-3

[2] Hoffman, R., Prchal, J.T., Samuelson, S., Ciurea, S.O. and Rondelli, D. (2007) Philadelphia Chromosome-Negative Myeloproliferative Disorders: Biology and Treatment. Biology of Blood and Marrow Transplantation, 13, 64-72.

https://doi.org/10.1016/j.bbmt.2006.11.003

[3] Scott, L.M., Beer, P.A., Bench, A.J., Erber, W.N. and Green, A.R. (2007) Prevalence of JAK2 V617F and Exon 12 Mutations in Polycythaemia Vera. British Journal of Haematology, 139, 511-512. https://doi.org/10.1111/j.1365-2141.2007.06806.x

[4] Vannucchi, A.M., Antonioli, E., Guglielmelli, P., et al. (2007) Prospective Identification of High-Risk Polycythemia Vera Patients Based on JAK2 ${ }^{\mathrm{V} 617 \mathrm{~F}}$ Allele Burden. Leukemia, 21, 1952-1959. https://doi.org/10.1038/sj.leu.2404854

[5] Rollison, D.E., Howlader, N., Smith, M.T., Strom, S.S., Merritt, W.D., Ries, L.A., Edwards, B.K. and List, A.F. (2008) Epidemiology of Myelodysplastic Syndromes and Chronic Myeloproliferative Disorders in the United States, 2001-2004, Using Data from the NAACCR and SEER Programs. Blood, 112, 45-52. https://doi.org/10.1182/blood-2008-01-134858

[6] Shuvaev, V., Martynkevich, I., Abdulkadyrova, A., et al. (2014) Ph-Negative Chronic Myeloproliferative Neoplasms-Population Analysis, a Single Center 10-Years' Experience. Blood, 124, 55-56. https://doi.org/10.1182/blood.V124.21.5556.5556

[7] Tefferi, A. and Barbui, T. (2019) Polycythemia Vera and Essential Thrombocythemia: 2019 Update on Diagnosis, Risk-Stratification and Management. American Journal of Hematology, 94, 133-143.

https://doi.org/10.1002/ajh.25303

[8] James, C., Ugo, V., Le Couedic, J.P., et al. (2005) A Unique Clonal JAK2 Mutation Leading to Constitutive Signalling Causes Polycythaemia Vera. Nature, 434, 1144-1148. https://doi.org/10.1038/nature03546

[9] Lippert, E., Boissinot, M., Kralovics, R., et al. (2006) The JAK2-V617F Mutatio Is Frequently Present at Diagnosis in Patients with Essential Thrombocythemia and Polycythemia Vera. Blood, 108, 1865-1867. https://doi.org/10.1182/blood-2006-01-013540

[10] Campbell, P.J., Scott, L.M., Buck, G., et al. (2005) Definition of Subtypes of Essential Thrombocythemia and Relation to Polycythaemia Vera Based on JAK2 V617F Mutation Status: A Prospective Study. Lancet, N366, 1945-193. https://doi.org/10.1016/S0140-6736(05)67785-9

[11] Kralovics, R., Guan, Y. and Prchal, J.T. (2002) Acquired Uniparental Disomy of Chromosome 9p Is a Frequent Stem Cell Defect in Polycythemia Vera. Experimental Hematology, 30, 229-236. https://doi.org/10.1016/S0301-472X(01)00789-5

[12] Huang, L.J., Constantinescu, S.N. and Lodish, H.F. (2001) The N-Terminal Domain of Janus Kinase 2 Is Required For Golgi Processing and Cell Surface Expression of Erythropoietin Receptor. Molecular Cell, 8, 1327-1338. https://doi.org/10.1016/S1097-2765(01)00401-4

[13] Ugo, V., Marzac, C., Teyssandier, I., Larbret, F., Lécluse, Y., Debili, N., Vainchenker, W. and Casadevall, N. (2004) Multiple Signaling Pathways Are Involved in Erythropoietin-Independent Differentiation of Erythroid Progenitors in Polycythemia Vera. Experimental Hematology, 32, 179-187. https://doi.org/10.1016/j.exphem.2003.11.003

[14] Mnjoyan, Z., Yoon, D., Li, J., et al. (2006) The Effect of the JAK2 V617F Mutation of PRV-1 Expression. Haematologica, 91, 411-412.

[15] Baxter, E.J., Scott, L.M., Campbell, P.J., et al. (2005) Acquired Mutation of the Tyrosine 
Kinase JAK2 in Human Myeloproliferative Disorders. The Lancet, 365, 1054-1061. https://doi.org/10.1016/S0140-6736(05)71142-9

[16] Prchal, J. (2006) Does JAK2 1849G>T Initiate Polycythemia Vera? Blood, 108, 8-9. https://doi.org/10.1182/blood-2006-04-016238

[17] Kralovics, R., Passamonti, F., Buser, A.S., et al. (2005) A Gain-of-Function Mutation of JAK2 in Myeloproliferative Disorders. The New England Journal of Medicine, 352, 1779-1790. https://doi.org/10.1056/NEJMoa051113

[18] Popat, U., Frost, A., Liu, E., Guan, Y.L., Durette, A., Reddy, V. and Prchal, J.T. (2006) High Levels of Circulating CD34 Cells, Dacrocytes, Clonal Hematopoiesis, and $J A K 2$ Mutation Differentiate Myelofibrosis with Myeloid Metaplasia from Secondary Myelofibrosis Associated with Pulmonary Hypertension. Blood, 107, 3486-3488. https://doi.org/10.1182/blood-2005-08-3319

[19] Onida, F., Ball, G., Kantarjian, H.M., et al. (2002) Characteristics and Outcome of Patients with Philadelphia Chromosome Negative, $B c r / A b l$ Negative Chronic Myelogenous Leukemia. Cancer, 95, 1673-1684. https://doi.org/10.1002/cncr.10832

[20] Tefferi, A. and Vardiman, J.M. (2008) Classification and Diagnosis of Myeloproliferative Neoplasms: The 2008 World Health Organization Criteria and Point-of-Care Diagnostic Algorithms. Leukemia, 22, 14-22. https://doi.org/10.1038/sj.leu.2404955

[21] Landgren, O., Goldin, L.R., Kristinsson, S.Y., Helgadottir, E.A., Samuelsson, J. and Björkholm, M. (2008) Increased Risks of Polycythemia Vera, Essential Thrombocythemia, and Myelofibrosis among 24577 First-Degree Relatives of 11039 Patients with Myeloproliferative Neoplasms in Sweden. Blood, 112, 2199-2204. https://doi.org/10.1182/blood-2008-03-143602

[22] Moulard, O., Mehta, J., Fryzek, J., Olivares, R., Iqbal, U. and Mesa, R.A. (2014) Epidemiology of Myelofibrosis, Essential Thrombocythemia, and Polycythemia Vera in the European Union. European Journal of Haematology, 92, 289-297. https://doi.org/10.1111/ejh.12256

[23] Vannucchi, A.M., Antonioli, E., Guglielmelli, P., Pardanani, A. and Tefferi, A. (2008) Clinical Correlates of JAK2V617F Presence or Allele Burden in Myeloproliferative Neoplasms: A Critical Reappraisal. Leukemia, 22, 1299-1307. https://doi.org/10.1038/leu.2008.113

[24] Hemminki, K., Zhang, H., Sundquist, J. and Bermejo, J.L. (2009) Myeloproliferative Disorders in Sweden: Incidence Trends and Multiple Tumors. Leukemia Research, 33, e14-e16. https://doi.org/10.1016/j.leukres.2008.04.014

[25] Lim, Y., Lee, J.-O. and Bang, S.-M. (2016) Incidence, Survival and Prevalence Statistics of Classical Myeloproliferative Neoplasm in Korea. Journal of Korean Medical Science, 31, 1579-1585. https://doi.org/10.3346/jkms.2016.31.10.1579

[26] Phekoo, K.J., Richards, M.A., Moller, H. and Schey, S.A. (2006) The Incidence and Outcome of Myeloid Malignancies in 2,112 Adult Patients in Southeast England. Haematologica, 91, 1400-1404.

[27] Arber, D.A., Orazi, A. and Hasserjian, R. (2016) The 2016 Revision to the World Health Organization Classification of Myeloid Neoplasms and Acute Leukemia. Blood, 127, 2391-2405. https://doi.org/10.1182/blood-2016-03-643544

[28] Barosi, G., Bergamaschi, G., Marchetti, M., et al. (2007) JAK2 V617F Mutational Status Predicts Progression to Large Splenomegaly and Leukemic Transformation in Primary Myelofibrosis. Blood, 110, 4030-4036. https://doi.org/10.1182/blood-2007-07-099184

[29] Jensen, M.K., De Nully Brown, P., Nielsen, O.J. and Hasselbalch, H.C. (2000) Inci- 
dence, Clinical Features and Outcome of Essential Thrombocythaemia in a Well-Defined Geographical Area. European Journal of Haematology, 65, 132-139. https://doi.org/10.1034/j.1600-0609.2000.90236.x

[30] Vardiman, J.W., Thiele, J., Arber, D.A., et al. (2009) The 2008 Revision of the World Health Organization (Who) Classification of Myeloid Neoplasms and Acute Leukemia: Rationale and Important Changes. Blood, 114, 937-951.

https://doi.org/10.1182/blood-2009-03-209262 